\title{
Electrochemical etching technique for neutron dosimetry
}

\author{
Ravi Chand Singh ${ }^{1 *}$, Manwinder Singh ${ }^{2}$ and HS Virk ${ }^{3}$ \\ ${ }^{1}$ Department of Physics, Guru Nanak Dev University, Amritsar-143 005, Punjab, India \\ ${ }^{2}$ Chemistry Division, Irrigation \& Power Research Institute, Amritsar-143 001, Punjab, India \\ ${ }^{3} 360$, Sector 71, Mohali-160 071, Punjab, India \\ E-mail : ravichand.singh@gmail.com
}

\begin{abstract}
In the present work we have employed allyl diglycol carbonate (CR-39) and cellulose triacetate (CTA) plastic for detection of neutron recoil tracks without radiator. For CR-39, the results reveal that registration efficiency is a function of duration of chemical pre-etching and the best results are obtained with chemical pre-etching of 3 hours. It was also investigated that the ac field strength of $28 \mathrm{kV} / \mathrm{cm}$ having $2.5 \mathrm{kHz}$ frequency was optimum for revelation of tracks. Interestingly the sensitivity is fluence dependent and it was constant up to a fluence of about $10^{8} \mathrm{n} / \mathrm{cm}^{2}$. The sensitivity abruptly decreased with increased fluence. At optimum experimental conditions the minimum detection limit for CR-39 was found to be $0.47 \mathrm{mSv}$. For CTA, the tracks have been revealed by electrochemical etching (ECE) only and the minimum detection limit was found to be $0.85 \mathrm{mSv}$ at optimum experimental parameters.
\end{abstract}

Keywords : Electrochemical etching, neutron dosimetry, CR-39, CTA.

PACS Nos. : 87.53.Pb, Qc

\section{Introduction}

The dosimetric application of the Electrochemical Etching (ECE) technique, with both internal and external radiator methods, seems to be more promising than the use of conventional chemical track etching techniques especially in case of low track densities. An unmatched advantage of ECE is the possibility of amplifying the nuclear tracks to voluminous discharge spots, thus the detection becomes considerably simpler, faster and more reliable than with chemical etching technique.

The credit for the first effort for the practical application of ECE technique in dosimetry goes to Sohrabi and co-workers $[1,2]$. Their studies were related with routine and emergency fast-neutron dosimetry. Following them, several laboratories have started the investigations to establish the credibility of technique for neutron dosimetric applications [3].

Various combinations of track etch neutron detectors with different external and 
internal radiators of heavily ionizing nuclear particles (fission fragments, recoils, alpha particles) have been developed. In most of the investigations PC (polycarbonate) plastic detector has been used extensively [4,5]. Other plastic track detectors such as cellulose triacetate, cellulose nitrate and polyethylene terephthalate sheets have also been used as well $[4,6]$. The present study reveals that CR-39 is more competent for this purpose.

\section{Experimental details}

In the present work we have employed CR-39 and CTA plastic sheets for detection of neutron recoil tracks. Samples of these plastic sheets were irradiated with beams of Am-Be neutron source. During irradiation of foils, no external proton radiator was used. The irradiation of CTA plastic sheets was also carried out with neutrons from ${ }^{252} \mathrm{Cf}$ source. For revelation of neutron induced tracks in CTA and CR-39 sheets, ECE and combination of chemical etching (CE) and ECE technique have been employed, respectively.

\subsection{Procedure for CR-39 :}

To find the optimum condition for the revelation of neutron induced tracks by ECE technique, we have followed the procedure involving the following two steps :

(a) As a first step, chemical etching of CR-39 foils was carried out with $6 \mathrm{~N} \mathrm{NaOH}$ at $70^{\circ} \mathrm{C}$ for various time intervals and thickness measurements were taken after every etching interval. With the result of optimum pre-etching the short-range alpha tracks produced by the natural radioactivity of air (natural background) and other surface inhomogeneities were over etched and appeared in track form of rounded tip unsuited to a subsequent ECE. In this way the background would be considerably reduced [7].

(b) As a second step, the same samples were etched electrochemically keeping the irradiated side in touch with same etching solution as used for pre-etching, but at the room temperature.

To optimize electrical parameters, experiments were performed at various ac field strengths and frequencies using specially designed ECE cell.

\subsection{Procedure for CTA :}

As CTA plastic is insensitive to alphas (responsible for background tracks) produced by natural radioactivity, the neutron irradiated sheets of this plastic were electrochemically etched without pre-etching. The optimum etching parameters for CTA being a field strength of $26 \mathrm{kV} / \mathrm{cm}$ at a frequency of $75 \mathrm{~Hz}$ using $6 \mathrm{~N} \mathrm{NaOH}$ solution as etchant at $25^{\circ} \mathrm{C}$ for $5 \mathrm{hr}$ [8].

Density of electrochemically etched tracks in CR-39 and CTA detectors was determined using optical microscope. 


\section{Results and discussion}

\subsection{Effect of pre-etching on track density in CR-39 :}

The variation of registration efficiency as a function of pre-etching time is represented in Figure 1. It is evident from the figure that a pre-etching time of $3 \mathrm{hr}$ gives the best registration efficiency. The results show that at optimum pre-etching time the thickness of layer removed from the single surface of detector is $4.5 \mu \mathrm{m}$. ECE background after $3 \mathrm{hr}$ pre-etching was $540 \pm 30$ tracks $/ \mathrm{cm}^{2}$.

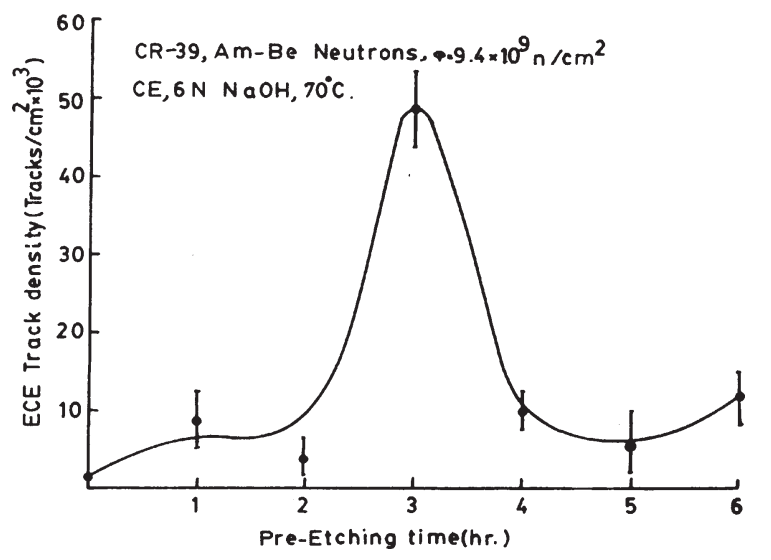

Figure 1. The fast neutron track density as a function of pre etching time in CR-39.

\subsection{Effect of electrical parameters :}

The curve shown in Figure 2 represents variation of track density verses ac field strength when CR-39 sheets pre-etched for $3 \mathrm{hr}$ were subjected to ECE using $6 \mathrm{~N}$ $\mathrm{NaOH}$ at $30^{\circ} \mathrm{C}$ at a fixed frequency of $2 \mathrm{kHz}$. The variation shows that ECE spot density rises linearly with field strength up to $28 \mathrm{kV} / \mathrm{cm}$. But further increase of field

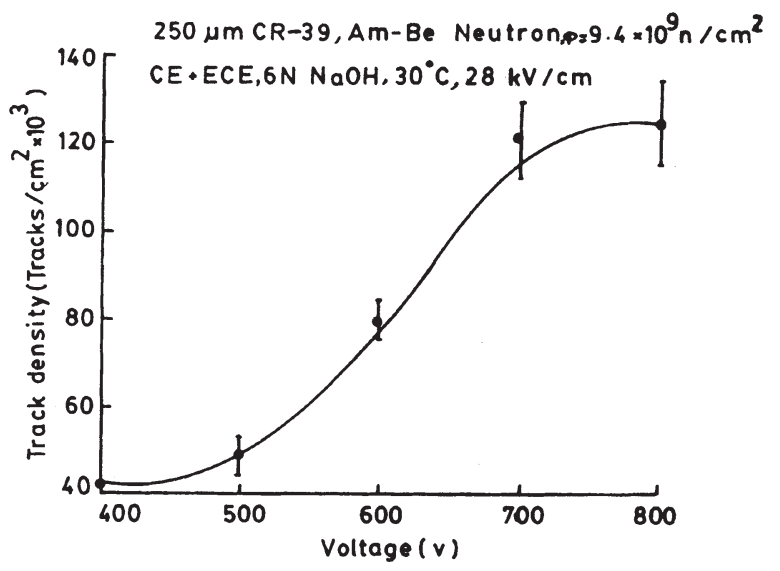

Figure 2. The variation of fast neutron track density with ac voltage in CR-39. 
gradient has no appreciable effect on the registration efficiency (Figure 2). On the other hand, at high voltages distortion of track-shape took place which is clearly shown in Figure 3. Field strength of $28 \mathrm{kV} / \mathrm{cm}$ was employed for further investigations.

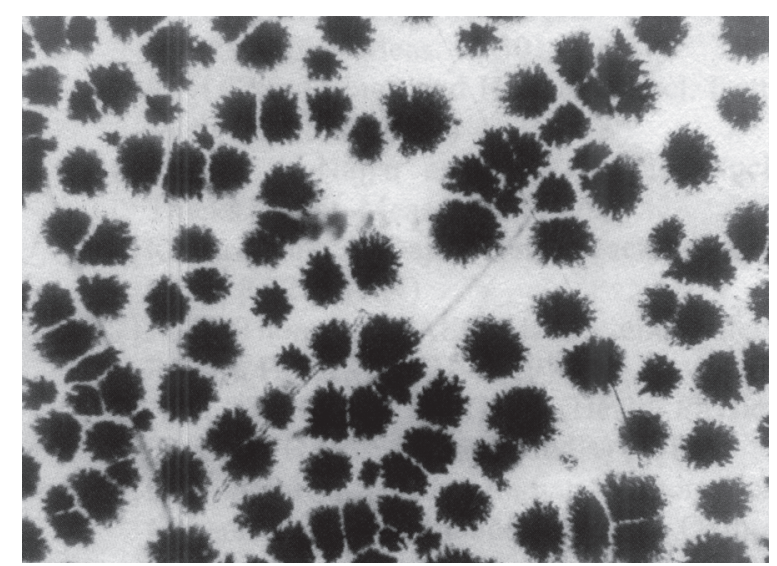

Figure 3. Microphotograph showing fast neutron recoil track-shapes in CR-39 when ECE was carried out after pre-etching : $32 \mathrm{kV} / \mathrm{cm}, 2 \mathrm{kHz}, 6 \mathrm{~N} \mathrm{NaOH}, 30^{\circ} \mathrm{C}$.

Figure 4 shows a curve representing variation of ECE spot density of neutron induced tracks verses alternating field frequency. The registration efficiency shows maxima around $2 \mathrm{kHz}$, which is due to the resonance type phenomenon exhibited by the detector during ECE [9]. The resonance frequency depends upon the type of the detector.

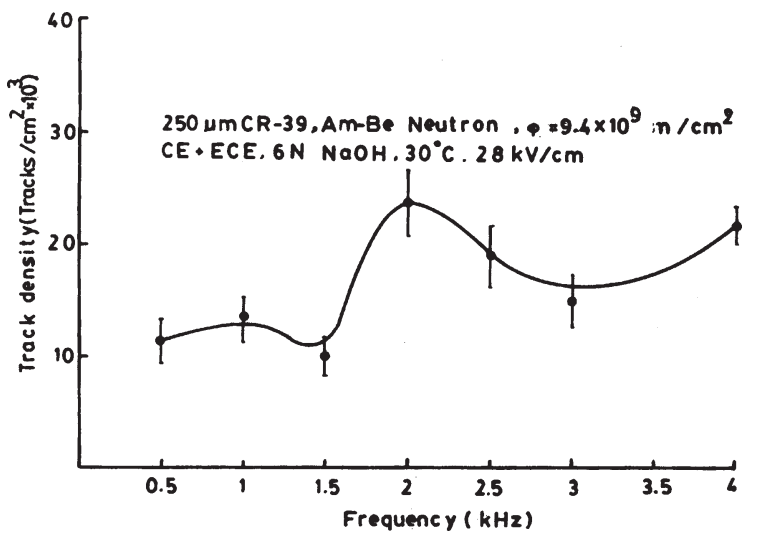

Figure 4. Fast neutron track density as a function of frequency in CR-39.

\subsection{Effect of fluence :}

All track-etched detectors exhibit a peculiar property of their response dependence on fluence, especially during ECE. This is because the ECE track size increases laterally. It has been reported that ECE response is a function of track density [10]. When the 
track density is too high the electrostatic repulsion forces between nearby tracks decreases the probability of the production of discharge spot during ECE. Therefore, it is expected that the sensitivity depends on the neutron fluence as well. Similar type of behavior has been observed for the CR-39 with Am-Be neutrons as shown in Figure 5. It is clear from the figure that sensitivity remains constant up to a fluence of about $10^{8} \mathrm{n} / \mathrm{cm}^{2}$, and then there is an abrupt decrease With the further increase of neutron fluence. The results obtained are in accordance with those reported elsewhere [11].

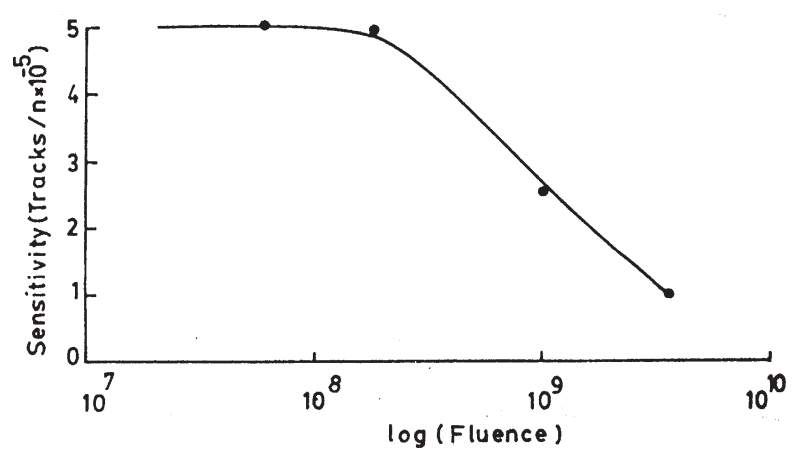

Figure 5. Sensitivity of CR-39 sheets to the (Am-Be) fast neutrons as a function of the neutron fluence, when using $(\mathrm{CE}+\mathrm{ECE})$ technique. $\mathrm{EC}=6 \mathrm{~N} \mathrm{NaOH}, 70^{\circ} \mathrm{C}, 3 \mathrm{hr} . \mathrm{ECE}=6 \mathrm{~N} \mathrm{NaOH}, 30^{\circ} \mathrm{C}, 28 \mathrm{k} \mathrm{V} / \mathrm{cm}, 2 \mathrm{k} \mathrm{Hz}, 2 \mathrm{hr}$.

\section{Dosimetric results}

Fast neutron dosimetric results obtained for neutron dose of $1.8 \times 10^{8} \mathrm{n} / \mathrm{cm}^{2}$ during ECE of CR-39 at optimized conditions are given below. If ' $D_{m}$ ' denotes the minimum detection limit of dose $(D)$ for which the fractional error $E(=\sigma D / D)$ is 0.5 , i.e. $50 \%$, then $D_{m}$ is given by [12] :

$$
D_{m}=2(1+\sqrt{1+2 B}) / K
$$

where $B$ is background track density and $K$ is the constant of proportionality (or sensitivity figure for a system, in our case it is registration efficiency) [13].

In our study the fluence $\Phi=1.8 \times 10^{8} \mathrm{n} / \mathrm{cm}^{2}$. Taking $1 \mathrm{n} / \mathrm{cm}^{2}=3.51 \times 10^{-10}$ Sv $[13,14]$ and $K=\left(\right.$ Tracks $\left./ \mathrm{cm}^{2}\right) /$ Sv.

\section{$D_{m}$ for CR-39}

Tracks $/ \mathrm{cm}^{2}=9027 \pm 5.7 \%$

$B=540 \pm 30$ tracks $/ \mathrm{cm}^{2}$

Gives $K=142877 \pm 5.7 \%$

From relation (1), $D_{m}=0.47 \mathrm{mSv}$

\section{$D_{m}$ for CTA}

Tracks $/ \mathrm{cm}^{2}=6770 \pm 3.9 \%$ 
$B=1000 \pm 50$ tracks $/ \mathrm{cm}^{2}$

Gives $K=107154 \pm 3.9 \%$

From relation (1), $D_{m}=0.85 \mathrm{mSv}$

The present study reveals that even though CR-39 is more sensitive than CTA any of these plastic track detectors may be employed for dosimetric applications depending upon the situational requirements.

\section{References}

[1] M Sohrabi and K Becker Nucl. Instr. Meth. 104409 (1972)

[2] M Sohrabi Hlth. Phys. 27598 (1974)

[3] K Turek and G Somogyi Proc. Int. Conf. on New Methods of Personnel Dosimetry (Czechoslovakia : Hradec Kralove) p274 (1977)

[4] G Somogyi, G Dajko, K Turek and F Spumy Nucl. Tracks 3125 (1979)

[5] M Sohrabi Nucl. Tracks 4131 (1981)

[6] A G Ramli and S A Durrani Proc. XI Int. Conf. on SSNTD (UK : Bristol) p237 (1981)

[7] G M Haasib, J W M Tuyn and J Dutrannois Nucl. Instr. Meth. 14163 (1977)

[8] Ravi Chand Singh and H S Virk Nucl. Tracks Radiat. Meas. 15301 (1988)

[9] Ravi Chand Singh and H S Virk Nucl. Instr. Meth. B30 598 (1987)

[10] S A R Al-Najjar and S A Durrani Nucl. Tracks Radiat. Meas. 899 (1979)

[11] K Turek and G Dajko Nucl. Tracks Radiat. Meas. 8121 (1984)

[12] J W Thomas USAEC Health and Safety Report, HASL-TM-71-1 (1971)

[13] A M Bhagwat $P h D$ Thesis (Health Physics Division, BARC, Bombay, India) (1983)

[14] H Kiefer and R Maushart Radiation Protection Measurement (Oxford : Pergamon Press) (1972) 